\title{
Reformation of Particle Filters in Simultaneous Localization And Mapping Problems
}

\author{
Masahiro Tanaka \\ Department of Information Science and Systems Engineering, Konan University \\ 8-9-1 Okamoto, Higashinada-ku, Kobe 658-8501, Japan
}

\begin{abstract}
SLAM is a hot topic in robotics community. It uses range sensors and aquires the distances to various directions as the sensor moves and changes its direction, so that it can acquire the environmental landscape and estimate the sensor's position/angle simultaneously. In this paper, we will explain the detail of FastSLAM by Montemerlo, and propose a modification of the algorithm.
\end{abstract}

\section{Introduction}

Simultaneous localization and mapping (SLAM) problem is an important problem in autonomous moving robotics, where acquiring the environment map and its trajectory are simultaneously aimed to be achieved. We have been developing a SLAM system on a pushcart, which is equipped with a laser sensor. Our goal is to use a senior car (an electric car for old person that moves slowly). It is not hard to find any objects that can be detected in one scanned data, since the nature of the data is very suitable for this problem. However, to know the location of the sensor position is a very hard problem, since we have to know its position only from the obtained scanned data. The importance of SLAM problem can be identified by considering the situation when we got lost our way in a maze. If we bring such equipment to a cave that is not explored sufficiently, it would be of great help.

The framework of SLAM can be described as follows.

1. Input the one cycle of scanned data. The data is a collection of points with a certain angle interval.

2. The obtained point positions must be rotated and moved by the inverse way of the sensor movement and rotation. However, we lack the knowledge of sensor movement and rotation information.

3. Adjust the observed points to the map that was built by using the past observation points. This new set of data may contribute adding some new part of the map.

4. Repeat the steps 1-3.

There are several strategies for estimating the sensor position and orientation from the observed
Table 1: Specification of SICK LMS291.

\begin{tabular}{|l|l|}
\hline Range & $8 \mathrm{~m} / 80 \mathrm{~m}$ \\
\hline Measurement resolution & $1 \mathrm{~mm} / 1 \mathrm{~cm}$ \\
\hline Scanning angle & $100^{\circ} / 180^{\circ}$ \\
\hline Angular resolution & $0.25^{\circ} / 0.5^{\circ} / 1^{\circ}$ \\
\hline System error & $\pm 35 \mathrm{~mm} / \pm 5 \mathrm{~cm}$ \\
\hline Scan time sequence & $53 \mathrm{~ms} / 26 \mathrm{~ms} / 13 \mathrm{~ms}$ \\
\hline Trasfer rate & $9.6 \mathrm{k} \sim 500 \mathrm{k}$ bps \\
\hline Interface & $\mathrm{RS}-232 \mathrm{C} / \mathrm{RS}-422$ \\
\hline
\end{tabular}

data, namely, (1) ICP(Iterative closest point)-based method, (2) PF(Particle Filter)-based method, and (3) KF(Kalman Filter)-based method.

In ICP algorithm, the new data points are given correspondence to the reference data set, where the policy of adjusting them is by the closest point to the reference one. The change of the position and the angle of the sensor can be obtained as a result of point matching. This method is a "passive" method. Kalman filterbased method is to estimate the position and angle of the sensor as the state variable. Particle filter-based method is to generate the candidates of the sensor position and the angle due to the dynamic model, and the generated candidates are validated by using the observed data. This method is an "active" method.

Comparing these methods, the ICP-based algorithm has a limit as the algorithm. The nearest point is not always the corresponding point. In many cases, the corresponding points are beyond several points ahead.

Hence we have been using the particle-filter-based method. To apply this, the core part of the algorithm consists of the following points.

1. building the dynamic model

2. dealing with the outlier or new points

\section{Sensor Property and Observation Data}

\subsection{Laser Range Sensor}

In our project, we are using a SICK LMS291 laser range sensor for sensing the environments. It measures the distances to the surface of objects in the surrounding by evaluating the travel time of an emitted laser 


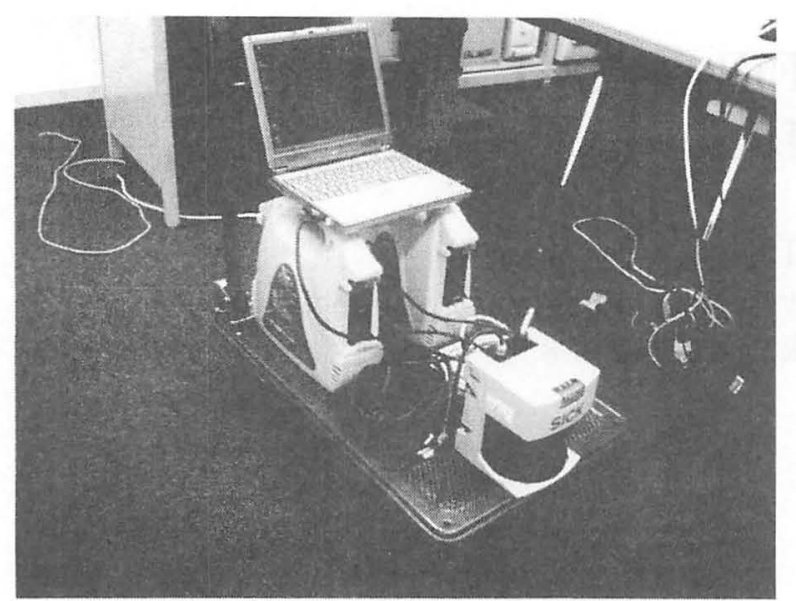

Fig. 1: Our laser range sensor: SICK LMS291 on a pushcart.

impulse. Note that this sensor is very common in the robotics fields.

As the property of this sensor, it is able to scan objects with angles of 0 to 180 degrees with selectable angular resolutions (0.25, 0.5 and 1.0 degrees), the measurable range is up to 30 meters. Table 1 shows the specification of the SICK LMS291 laser range sensor.

We are now doing experiments using a carrying cart instead of the wheelchair or pushcart for elderly persons. Figure 1 shows the sensor on the cart.

\subsection{Data Specification}

The laser sensor rotates about 40 times/second. The angle resolution can be set to $0.25,0.5$ or 1 degrees. Here we are using 0.5 degree. The observation view can be set to 100 or 180 degree, and we have set it to 180 degree. We suppose that the sensor is located on a flat place, and its posture is completely characterized by its position $(x, y)$ and the horizontal angle $\theta$. Hence the sensor information is denoted by a set $\{x, y, \theta\}$.

Distances to reflecting objects in a view of the sensor can be denoted as

$$
\left\{r_{i}, i=0, \ldots, 360\right\}
$$

whose position in local $(x, y)$ coordinate is

$\left(p_{i}, q_{i}\right)=\left(r_{i} \cos \left(\frac{\pi}{360} i\right), r_{i} \sin \left(\frac{\pi}{360} i\right)\right), i=0, \ldots, 360$

The precision of the data is recorded by $\mathrm{mm}$, and the observable maximum length is $8 \mathrm{~m}$ (the value is 8000 ), however, if some object is observed farther than this, the value is recorded as 8183 . Hence we should deal with the instance as a missing data or unobservable far point if this value is detected.

The observation is done while sensor is rotating. So, the data in the same scan is not observed at a same time. Here we set the time unit as the time the sensor rotates the unit angle (i.e. 0.5 degree). Then we have the observation data as

$$
\begin{gathered}
(r(t), \alpha(t)), t=0, \ldots, 360 \\
\alpha(t)=t \pi / 360(\text { radian })
\end{gathered}
$$

or

$$
(p(t), q(t)), i=0, \ldots, 360
$$

\section{Relation between Local and World Coordinates}

Before discussing the SLAM problem in detail, here we briefly see some fundamental elements of the problem.

We define the sensor position as $(x, y)$, and the angle $\theta$ ) by the world coordinate. Suppose the world coordinate is defined in some rigid way. Consider a point $\mathrm{P}(a, b)$ defined in the world coordinate.

For a simple discussion, the initial value $(x, y, \theta)=$ $(0,0,0)$.

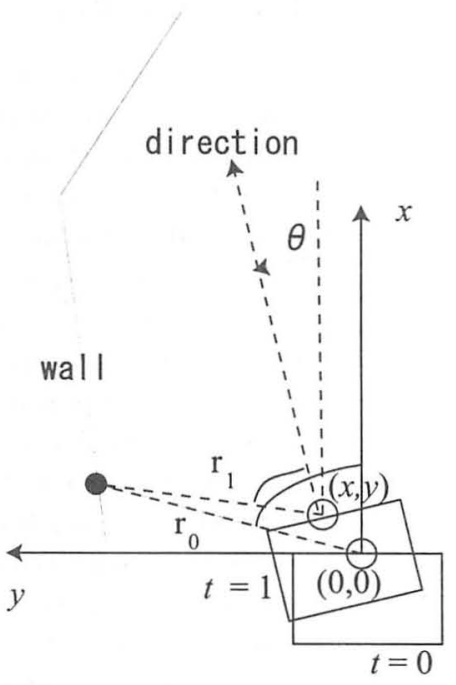

Fig. 2: Geometrical explanation of variables.

Suppose a landmark denoted by a black dot can be measured. However, the sensor position cannot be determined by one observation point. Actually, at least 3 points are needed to be observed.

From the fundamental consideration of geometry of this case, we have the nonlinear equation between the observation value $r$ and $\alpha$ and the world coordinate of the sensor information $x, y, \theta$ as

$$
\left(\begin{array}{l}
a \\
b
\end{array}\right)=\left(\begin{array}{c}
\cos (\theta+\alpha) \\
\sin (\theta+\alpha)
\end{array}\right) r+\left(\begin{array}{l}
x \\
y
\end{array}\right)
$$

or, equivalently,

$$
\begin{aligned}
& r=\sqrt{(a-x)^{2}+(b-y)^{2}} \\
& \alpha=\tan ^{-1}\left(\frac{b-y}{a-x}\right)-\theta
\end{aligned}
$$




\section{FastSLAM}

FastSLAM is an algorithm by Montemerlo [1] for SLAM problem. It is a mathematical algorithm using particle filter [5].

\subsection{SLAM Problems}

SLAM problems in general are based on the posterior of the landmarks and the sensor information [1]

$$
p\left(s^{t}, \Phi \mid z^{t}, n^{t}\right)
$$

where the superscript $t$ denotes the accumulation of the variable from 1 to $t$, i.e.

$$
s^{t}=\{s(1), \cdots, s(t)\}
$$

further where $\Phi$ is the set of landmarks, $z(t)$ is the observation at time $t, n(t)$ is the association between the observation and the landmarks. In (4), association is assumed to be known.

It has been proved [7] that this probability density function (PDF) (4) is able to expressed as follows:

$$
p\left(s^{t}, \Phi \mid z^{t}, n^{t}\right)=p\left(s^{t} \mid z^{t}, n^{t}\right) \prod_{n=1}^{N} p\left(\phi_{n} \mid s^{t}, z^{t}, n^{t}\right)
$$

where the first factor in the right hand side is the path posterior, and the second ones are the landmark estimators.

Since $n^{t}$ is basically unknown in the ordinary SLAM problems, we should consider

$$
\begin{aligned}
p\left(s^{t}, \Phi \mid z^{t}\right) & =\sum_{n^{t}} p\left(s^{t}, \Phi \mid z^{t}, n^{t}\right) p\left(n^{t} \mid z^{t}\right) \\
& \sim p\left(s^{t}, \Phi \mid z^{t}, n^{t}\right)
\end{aligned}
$$

The approximation holds if the estimated association has a very large probability, and it is usually the case for a system where the sensor moves only a little in a step.

This factorization indicates that we should estimate

- the sensor pose that yields the highest probability when the observation and the association are given, and

- each of the landmark position so that it yields the highest probability when the observation and the association are given.

\subsection{Whole Algorithm}

FastSLAM maintains the landmarks and the trajectory of the sensor movement. The landmarks are the static salient points among the observed points. New landmarks are added to the set of landmarks, and the positions of them are updated as new observation is made.

The overall algorithm including FastSLAM can be written as follows.
Initialization Let $t=0$, and set the landmark set $\Phi(0)=\phi$, and set $s(0)=\left[\begin{array}{lll}0 & 0 & 0\end{array}\right]^{\prime}$.

do the following for time $t=1,2, \ldots$

(1) observe $r(t)$ and $\alpha(t)$.

(2) judge if the current point is a landmark. The algorithm for (2) will be described below.

Estimation of Landmark Position It is necessary for this value to be easily detected, and should be stable. In that respect, we decided to detect points such as the points on the corner, because they are easily seen from various angles and positions. Also, it is computationally very cheap.

This can be done in the local coordinate.

$$
P_{\theta}= \begin{cases}\text { feature point } & \text { condition1 } \\ \text { non-feature point } & \text { otherwise }\end{cases}
$$

where condition 1 is if $r(\theta-1)+r(\theta+1)-$ $2 \mathrm{r}(\theta)>\mathrm{D}$ and $\mathrm{r}_{\theta}<8000$. We empirically determined the value of $D$ as 200 .

Figures 3 and 4 may validate this discussion. The landmarks are stably detected, and the view does not change much in a single step.

(3) call FastSLAM $(S(t-1), r(t), \alpha(t))$

end of loop for $t$.

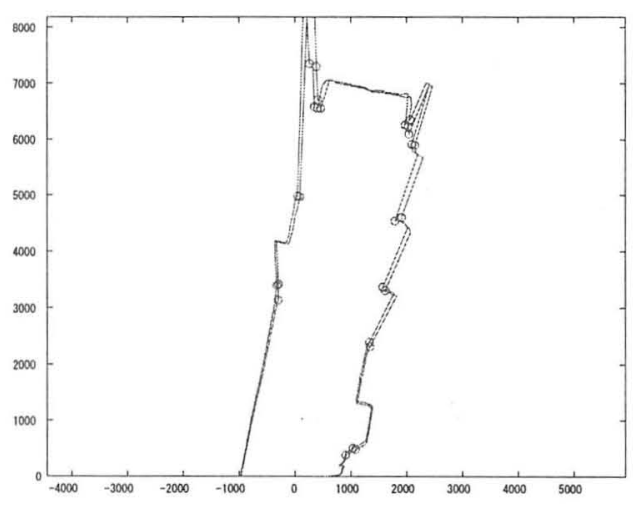

Fig. 3: time $=2$

\subsection{FastSLAM}

The fundamental flow of FastSLAM is as follows.

arguments $S(t-1), r(t), \alpha(t)$ : given (detected landmark)

for each particle $(1, \ldots, M)$ do the following.

(a) generate a new particle draw a new particle based on the dynamic model of the state vector. 


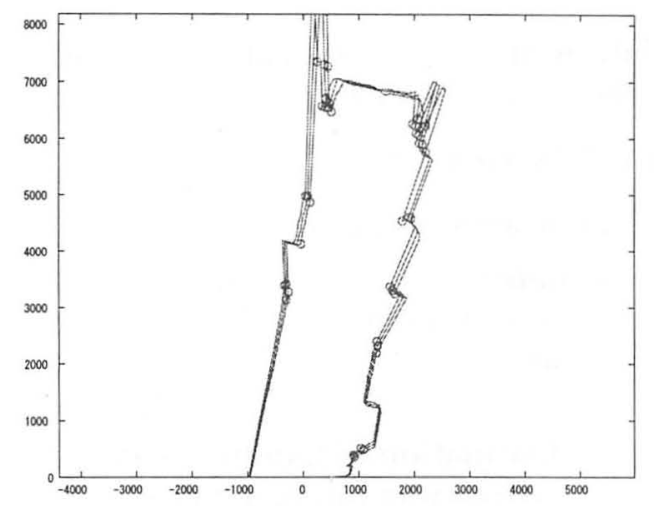

Fig. 4: time $=3$

(b) for each landmark in step $t-1$ do the following.

- (b-1) predict the distance and the angle (observation)

- (b-2) compute the likelihood based on the distance between the current landmark and each of the accumulated landmarks.

end loop

(c) find the correspondence based on the distances.

(d) if it is a new landmark create one based on the inverse function of the observation.

(e) if it is an existing landmark update the position of the landmark estimation.

other landmarks are left unchanged.

(f) calculate the weight of this particle

end loop

\subsection{Detail of Each Part of FastSLAM}

(a) Generating a new particle Particle filter is a simulation-based algorithm. The new particles are generated according to the stochastic model of the state evolution. The simplest model is the random walk

$$
s(t)=s(t-1)+w(t)
$$

where $s(t) \in \mathbf{R}^{3}$ is the state vector

$$
s(t)=[x(t) y(t) \theta(t)]^{\prime}
$$

$w(t)$ is also a 3 -dimensional small vector which gives the change of the sensor position and angle in the unit time.

At time $t$, one particle is generated from one particle at time $t-1$, i.e.

$$
s^{(m)}(t)=s^{(m)}(t-1)+w(t)
$$

where $w(t)$ is a random vector generated according to its prior PDF. (b-1) Estimation of Landmark Position Compute the predicted observation value (in local polar coordinate) by

$$
\hat{z}_{n}(t)=f\left(\mu_{n}^{m}(t-1), s^{(m)}(t)\right)
$$

using the relations (2) and (3), and the covariance matrix

$$
\begin{gathered}
Z_{n}(t)=G(t) \Sigma_{n}^{(m)}(t-1) G(t)^{\prime}+R(t) \\
G(t)=\nabla_{\mu_{n}} f\left(\mu_{n}, s(t)\right)
\end{gathered}
$$

(b-2) Likelihood In the FastSLAM algorithm, one landmark is selected as the corresponding point for the input feature point. The likelihood is the measure of goodness of the correspondence. It is given by

$$
\begin{gathered}
p_{n}^{(m)}(t)=\left|2 \pi Z_{n}(t)\right|^{-1 / 2} \times \\
\times \exp \left(-\frac{1}{2}\left(z(t)-\hat{z}_{n}(t)\right)\right)^{\prime} Z_{n}^{-1}(t)\left(-\frac{1}{2}\left(z(t)-\hat{z}_{n}(t)\right)\right)
\end{gathered}
$$

where

$$
\begin{gathered}
\hat{z}_{n}(t)=g\left(s^{(m)}, \mu_{n}^{(m)}(t-1)\right) \\
Z_{n}(t)=G_{n} \Sigma_{n}^{(m)}(t-1) G_{n}^{\prime}+R
\end{gathered}
$$

and $g(s, \mu)$ is the observation vector consisting of the distance and the angle, i.e.

$g(s, \mu)=\left[\begin{array}{c}r(s, \mu) \\ \alpha(s, \mu)\end{array}\right]=\left[\begin{array}{c}\sqrt{\left(\mu_{x}-s_{x}\right)^{2}+\left(\mu_{y}-s_{y}\right)^{2}} \\ \tan ^{-1}\left(\frac{\mu_{y}-s_{y}}{\mu_{x}-s_{x}}\right)-\theta\end{array}\right]$

and $G$ is the Jacobian given by

$$
\begin{gathered}
G=\left[\begin{array}{ll}
\frac{\partial r}{\partial \mu_{x}} & \frac{\partial r}{\partial \mu_{y}} \\
\frac{\partial \alpha}{\partial \mu_{x}} & \frac{\partial \alpha}{\partial \mu_{y}}
\end{array}\right] \\
=\left[\begin{array}{cc}
\frac{\mu_{x}-s_{x}}{\sqrt{q}} & \frac{\mu_{y}-s_{y}}{\sqrt{q}} \\
-\frac{\mu_{y}-s_{y}}{q} & \frac{\mu_{x}-s_{x}}{q}
\end{array}\right]
\end{gathered}
$$

where

$$
q=\sqrt{\left(\mu_{x}-s_{x}\right)^{2}+\left(\mu_{y}-s_{y}\right)^{2}}
$$

\subsubsection{Landmark Creation}

If $n(t)$ is a new feature point, create the landmark by the inverse of the estimate by

$$
\begin{gathered}
n(t)=N^{(m)}(t-1)+1 \\
\mu_{n(t)}^{(m)}(t)=g^{-1}\left(s^{(m)}(t), \hat{x}_{n(t)}(t)\right) \\
\Sigma_{n(t)}^{(m)}(t)=\left(G^{\prime} R^{-1} G\right)^{-1}
\end{gathered}
$$

\subsubsection{Update of Landmark Position}

$$
\begin{gathered}
N_{t}^{(m)}=N_{t-1}^{(m)} \\
K_{n(t)}(t)=\Sigma_{n(t)}^{(m)}(t-1) G^{\prime} Z_{n(t)}^{-1}(t) \\
\mu_{n(t)}^{(m)}(t)=\mu_{n(t)}^{(m)}(t-1)+K_{n(t)}(t)\left[z(t)-\hat{z}_{n(t)}(t)\right] \\
\Sigma_{n(t)}^{(m)}(t)=\left[I-K_{n(t)}(t) G_{n(t)}\right] \Sigma_{n(t)}^{(m)}(t-1)
\end{gathered}
$$




\subsubsection{Weight of the landmark}

This landmark has a weight according to the equation [1]

$$
\begin{aligned}
& w^{(m)}(t)=\frac{p\left(s^{(m)}(t) \mid z^{t}, n^{t}\right)}{p\left(s^{(m)}(t) \mid z^{t-1}, n^{t-1}\right)} \\
&=\frac{1}{\sqrt{\mid 2 \pi Z_{n(t)}(t)}} \times \\
& \times \exp \left(-\frac{1}{2}\left(z(t)-\hat{x}_{n(t)}(t)\right)^{\prime} Z_{n(t)}^{-1}(t)\left(z(t)-\hat{x}_{n(t)}(t)\right)\right)
\end{aligned}
$$

\subsection{Particle Selection Mechanism}

Particles are selected by resampling mechanism. The resampling is stochastically done by using the weight which is calculated by

$w^{(m)}(t)=\frac{\text { target distribution }}{\text { proposal distribution }}=\frac{p\left(s^{t,(m)} \mid z^{t}, n^{t}\right)}{p\left(s^{t,(m)} \mid z^{t-1}, n^{t-1}\right)}$

that reduces to a simple form as

$$
w^{(m)}(t)=p\left(z(t) \mid s^{t,(m)}, z^{t-1}, n^{t}\right)
$$

\section{Our Modification}

FastSLAM algorithm processes the detected landmarks (feature points) one by one where time index increases for one landmark. It is true that the sensor may change its position and angle when it detects the feature points, but the time intervals between the detected feature points are not uniform. The FastSLAM algorithm needs to add some description on this point. Moreover, the time when the sensor rotates angles from 180 degree to 0 degree must be taken into consideration.

Here we propose to deal with the observed feature points obtained in one scan simultaneously. The time instants of capturing feature points in one scan are not the same, but the time difference is neglectably small considering the slow change of the sensor posture.

The slow change also contributes to the model simplifications. In the original FastSLAM, the positions of the landmarks are estimated by using Extended Kalman Filter. However, we need to use a sophisticated model for this purpose. Instead, we use the original detected signals because the change in one cycle seems very small.

The overall algorithm can be written as follows.

\section{Proposed Algorithm}

Initialization Let $t=0$, and set the landmark set $\Phi(0)=\phi$, and set $s(0)=\left[\begin{array}{lll}0 & 0 & 0\end{array}\right]^{\prime}$.

do the following for time $t=1,2, \ldots$ (This time index increases due to the scan times.)

(1) observe one set of ranges for each angle.

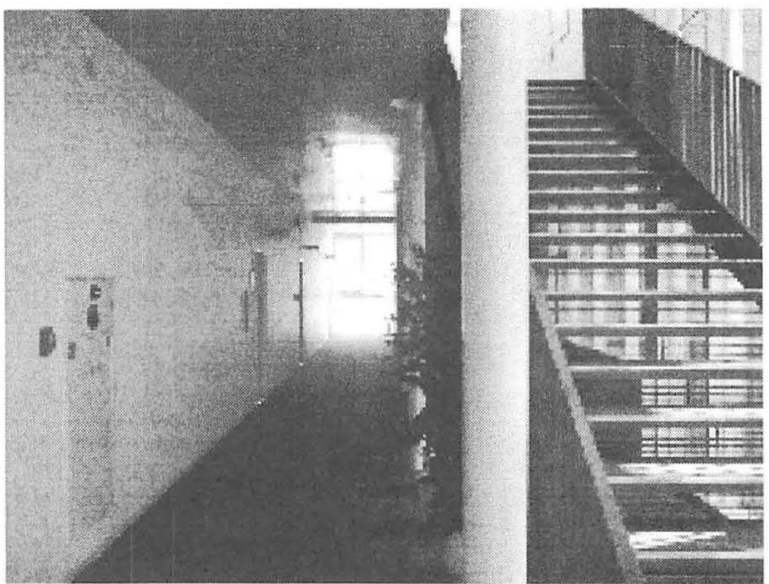

Fig. 5: Experimental environment

(2) detect feature points (candidates of landmarks).

(3) call $\operatorname{SLAM}\left(S(t-1), r_{i}(t), \alpha_{i}(t)\right) \quad((i=$ $0, \ldots, 360)$.

end of loop for $t$

The fundamental flow of SLAM is as follows.

arguments $S(t-1), r(t), \alpha(t)$ : given (detected landmark)

for each particle $(1, \ldots, M)$ do the following.

(a) generate a new particle draw a new particle based on the dynamic model of the state vector.

(b) for each landmark in the previous scan $t-1$ predict the distance and the angle (observation)

(c) for each landmark in the current scan $t$ find the correspondence among the existing landmark or define a new landmark, and compute the likelihood based on the distance between the current landmark set and each of the accumulated landmarks. The likelihood is given by

$$
\begin{gathered}
p^{(m)}(t)=\sum_{n}\left|2 \pi Z_{n}(t)\right|^{-1 / 2} \times \\
\times \exp \left(-\frac{1}{2}\left(z(t)-\hat{z}_{n}(t)\right)\right)^{\prime} Z_{n}^{-1}(t)\left(-\frac{1}{2}\left(z(t)-\hat{z}_{n}(t)\right)\right)
\end{gathered}
$$

end loop

(d) evolve the particles where good ones are copied, and bad ones are deleted. 


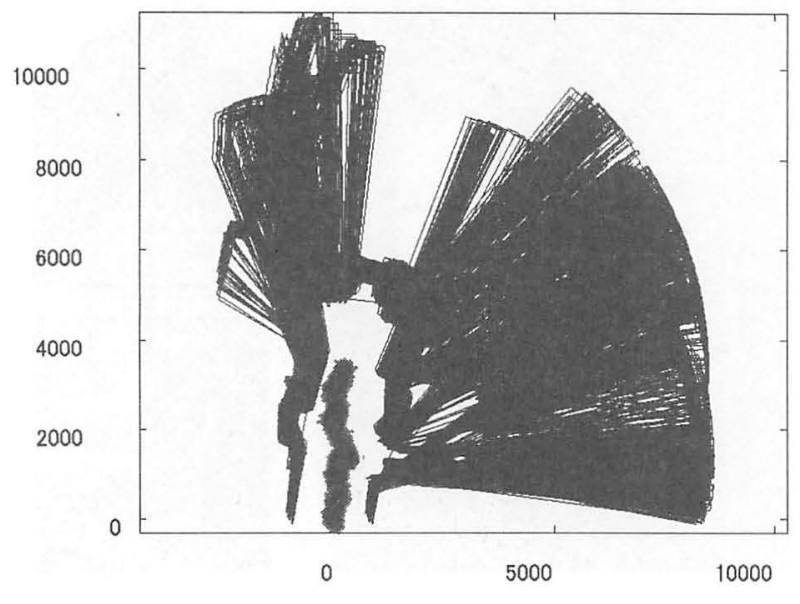

Fig. 6: Result of localization and automatically map building

\subsection{Experimental Result}

Here we will show an experimental result by applying particle filter for localization problem. Figure 5 shows the experimental environment for test of our localization algorithm. The red circles denote the estimated positions of the sensor. The green area are the collection of particle estimates. The blue line is the view from the sensor converted into the estimated world coordinate. As the sensor moves, the view changes. In this experiment, the number of particles of the particle filter was 1000 .

\section{Conclusions}

In this paper, we carried out a preliminary experiment to test the point based localization method in indoor environment, and provided the efficacy of matching method. Next, we implement a particle filter [2] of the localization algorithm. Particle filter yields good estimates of the nonlinear system. Particle filter uses multiple number of solution candidates, and evolved them based on the system model. Good solution candidates are employed to the origin of the update.

In our future work, we will improve the accuracy of localization algorithm and perform localization task for other situations. Experimental comparisons with other approaches should also be considered.

\section{Acknowledgment}

This work was supported in part by GRANT-IN-AID for ORC Project from the Japanese Government Structure for Education, Culture, Sports, Science and Technology.

\section{References}

[1] M. Montemerlo and S. Thrun: FastSLAM :A Scalable Method for Simultaneous Localization and Mapping Problem in Robotics, Springer, 2007.

[2] T. Higuchi, Monte Carlo Filter Using the Genetic Algorithm Operators, Journal of Statistical Computation and Simulation, Vol. 59, No. 1, pp. 1-23, 1997

[3] W. Rencken, Concurrent localisation and map building for mobile robots using ultrasonic sensors, Proceedings of the IEEE/RSJ International Conference on Intelligent Robots and Systems, pp. 21292197, 1993

[4] J. Selkäinaho, Adaptive Autonomous Navigation of Mobile Robots in Unknown Environments, Ph.D. thesis, Helsinki University of Technology, Finland, 2002

[5] B. Ristic, S. Arulampalam and N. Gordon, Beyond the Kalman Filter -Particle Filters for Tracking Applications-, Artech House, Boston, London, 2004

[6] S. Haykin (Ed.), Kalman Filtering and Neural Networks, Wiley Interscience, 2001

[7] K. Murphy, Bayesian map learning in dynamic environments, Advances in Neural Information Processing Systems (NIPS), MIT Press, 1999. 